\title{
Un corps professionnel renouvelé : les professeurs des universités québécoises francophones entre 1959 et 1976
}

\author{
PIERRE DANDURAND*
}

\section{ABSTRACT}

At the same time as a new concept of universities was being developed, during the post-war period, a new professionalisation project emerged among Quebec's Francophone university professors, placing scientific research at the centre of university practices which, in order to be accomplished, required changes in university structures.

This article attempts to show how, from 1959 onwards, these new conditions came about with more direct State funding of universities and very rapid institutional development along with the bureaucratization of the university system and unionization of professors.

By 1976, the end of the period covered by our study, the unionization of professors was achieved and the outline of the movement toward professionalisation characteristic of the 1980's already in place.

\section{RÉSUMÉ}

Parallèlement à une nouvelle conception de l'université, on a vu s'imposer dans l'après-guerre, chez les professeurs des universités francophones du Québec, un nouveau projet de professionnalisation qui plaçait la recherche scientifique au centre des pratiques universitaires et exigeait, pour se réaliser, des transformations dans les structures universitaires.

L'article tente de montrer comment ces nouvelles conditions se sont réalisées à partir de 1959 avec une prise en charge plus directe de l' université par l' Etat et un développement institutionnel très rapide qui s'est accompagné d' une bureaucratisation du système universitaire et d' une syndicalisation des professeurs.

Au moment où l'analyse se termine, en 1976, la syndicalisation des professeurs est chose faite et les grandes lignes du mouvement de qui professionnalisation caractérise les années 1980 sont déjà en place.

*Département de sociologie, Université de Montréal. 
Par avancées successives, l'enseignement des sciences et la recherche ont, à partir des années 1920 et surtout dans l'après-guerre, pris une place de plus en plus importante dans le système universitaire québécois. Cette valorisation de la rationalité scientifique comme mode de connaissance allait transformer le modèle culturel universitaire, y délogeant de leur position centrale la théologie et la philosophie scolastique pour une part et les savoirs professionnels (ceux des vieilles professions traditionnelles) d'autre part. On assiste du même coup, et dans le même mouvement, à plusieurs transformations du corps professoral, en lien avec ce changement dans la culture académique. Mais l'évolution du corps professoral dépend aussi de développements institutionnels, de la montée des populations étudiantes, et renvoie enfin à des changements plus profonds dans les structures sociales, notamment à l'émergence des nouvelles classes moyennes et à la constitution de l'État-providence.

Ainsi dès l'après-guerre, les conditions de la pratique de l'enseignement et de la recherche au niveau universitaire seront redéfinies. Le sens de cette évolution peut se résumer sous quelques titres : 1) d'abord, un double processus de sécularisation des savoirs et de décléricalisation du corps professoral; 2) en second lieu, un processus non terminé de professionnalisation des tâches des universitaires. L'ensemble de ces processus conduit à une autonomisation relative du corps professoral, d'abord vis-à-vis de l'Église catholique mais aussi plus largement vis-à-vis les agents de la société civile et en particular vis-à-vis les grandes corporations professionnelles. Cette autonomie relative reste cependant une autonomie problématique face à l'État-employeur et aussi face à une couche d'administrateurs universitaires, que le développement et la bureaucratisation du système ont créée.

Dans cet article ${ }^{1}$, notre analyse débute au moment où, à la fin des années 1950 , un nouveau projet de professionnalisation est déjà formulé mais sans que soient encore réunies les conditions de sa réalisation. Les mouvements de sécularisation des savoirs et de laïcisation du corps professoral sont alors irrémédiablement engagés. Par ailleurs, dans l'après-guerre, des clientèles de plus en plus nombreuses se pressent aux portes des universités. Le développement rapide du système universitaire provoque alors des situations financières par moments dramatiques et force un réaménagement des rapports de l'université avec le réseau des grandes institutions, en particulier l'État et l'Église mais aussi, et surtout en milieu anglophone, avec les entreprises philanthropiques, elles-mêmes plus ou moins liées au monde de l'économie. Pour le professeur laïc, qui voulait faire carrière, cette situation signifie le plus souvent une grande insécurité et des conditions de travail des plus austères. Le Rapport Massey (1951) et le Rapport Tremblay (1956) condamneront à tour de rôle cette situation. Il devient ainsi de plus en plus acquis qu'il faille asşurer à l'université des ressources financières suffisantes et stables et permettre du même coup aux professeurs laïcs de poursuivre décemment une carrière académique.

“...Si, écrit-on dans le Rapport Tremblay (1956, t. I, p. 254) pour un religieux, dont le régime de vie est défini et la sécurité matérielle garantie par une communauté, 
l'enseignement est une forme d'apostolat, pour le laïc, il est une profession, c'est-à-dire une manière de pourvoir par le travail à sa subsistance et à celle d'une famille et de réaliser le plus complètement possible sa vie. Ses garanties de stabilité, de sécurité, de pleine mise en valeur de lui-même que le religieux trouve d'abord dans son ordre, le lä̈ doit les trouver dans sa profession."

Les querelles sur les juridictions fédérales/provinciales dans le domaine de l'éducation vont bloquer jusqu'en 1959 cette question du financement par l'État des universités mais la légitimité d'un projet de carrière académique pour les laïcs est acquise. Par ailleurs, c'est au cours de cette période que prend vraiment forme un modèle nouveau de la profession universitaire que Léon Dion (1958) a formulé d'ailleurs de façon exemplaire. Les pratiques de ce nouvel universitaire, écrit Dion, devront être essentiellement guidées par "les normes scientifiques". Et c'est à partir des exigences de la science que le corps professoral devra de lui-même, de façon autonome, définir sa place et ses fonctions dans l'université. Ainsi le nouvel universitaire sera, souhaite-t-on, laïc et de la "race" des scientifiques.

C'est là le projet de professionnalisation qui s'est imposé de plus en plus au cours des années 1940 et 1950. Dans la mesure où la recherche constitue l'axe central de cette conception normative de la fonction universitaire, on y réfèrera comme à un modèle humboldtien, soit à une représentation de l'université dominée par les activités de recherche et où l'enseignement devient une préoccupation de second ordre (Blondin, 1987). Dans cette perspective humboldtienne, on considère même que le fait d'être habile chercheur garantit quasi de soi les qualités pédagogiques de l'universitaire. Ce modèle, élaboré dans les universités prussiennes du XIX ${ }^{\mathrm{e}}$ siècle, marque encore profondément la culture académique. Pour nous, il a une valeur heuristique : il permet d'établir dès le départ dans quel sens s'orientera le mouvement de professionnalisation du corps professoral québécois. Il aurait même pu nous permettre de prédire l'émergence de tensions entre la recherche et l'enseignement. Comme le souligne justement Gingras (1987, p. 153-156), ce modèle, quand on cherche à le réaliser, institue de lui-même cette tension entre ces deux grandes fonctions des universités. Effectivement, on verra que les débats qui accompagnent le développement du corps professoral tournent le plus souvent autour des places respectives à attribuer à l'enseignement et à la recherche.

Le construit symbolique qu'est ce projet de professionnalisation sera constamment un enjeu disputé, à l'intérieur même du groupe des professeurs, par différentes fractions. Il sera aussi un enjeu important dans les échanges entre les professeurs et les principaux agents du champ universitaire, administrateurs des universités, fonctionnaires du ministère de l'Éducation, gestionnaires des institutions para-publiques comme le Conseil des universités, le Conseil supérieur de l'éducation, la Crépuq. Il agira enfin comme guide et comme base de justification des stratégies des professeurs engagés dans les luttes pour assurer leur position dans le champ universitaire et plus largement dans la société. Les mouvements de professionnalisation sont en effet des luttes de classement et le projet professionnel n'est pas un idéal-type au sens wébérien du terme mais bien un projet à teneur idéologique comme Hughes (1971) a tenté de le montrer. 
Si le projet de professionnalisation est déjà bien en place au début des années 1960 , les deux conditions à une véritable institutionnalisation de la carrière d'universitaire, telles que posées dans le Rapport Tremblay de 1956, seront l'une et l'autre acquises au milieu des années 1970. Ces conditions impliquaient d'une part une source stable et importante de revenu pour les universités et, d'autre part, une reconnaissance juridique qui assure aux universitaires un statut comme corps autonome disposant de droits et de devoirs. Dans le premier cas c'est l'intervention de l'État, plus précisément une politique de subvention à l'enseignement supérieur, qui répondra à ce besoin de sécurité matérielle; dans le deuxième cas, ce sera, après la création d'associations de professeurs, le regroupement des enseignants dans des organisations syndicales. Le contexte institutionnel dans lequel s'insèrent les pratiques de recherche et d'enseignement des universitaires se trouveront ainsi modifées de façon majeure ${ }^{2}$. Si on ajoute qu'à la même période, les effectifs professoraux augmenteront de façon fulgurante, tout ceci nous amène à penser que c'est dans cet espace-temps que se constitue effectivement le "nouveau" corps professoral, soit un corps qui cherche à s'approprier les attributs valorisés d'une culture scientifique devenue dominante dans le champ universitaire.

\section{a) La période 1959-1976}

Cette période, qui marque le renouvellement du corps professoral, va donc en gros du début des années 1960 jusqu'au milieu des années 1970. Si on veut indiquer plus précisément les événements qui la balisent, il faut mentionner au départ ce qu'on a appelé la Grande charte de l'éducation de 1959. Dans cette charte, le gouvernement provincial convient entre autres de la nécessité d'accorder aux universités des subventions non plus discrétionnaires mais statutaires. Il se reconnaît ainsi formellement des responsabilités accrues dans le développement de l'enseignement supérieur. Par la suite se précisera et se structurera l'action de l'État provincial dans le champ universitaire, notamment, rappelons-le, avec la création du ministère de l'Éducation et sa Direction générale de l'enseignement supérieur (1964), avec la mise sur pied de l'Université du Québec (1969), et, enfin, par le biais d'un maquis d'institutions para-gouvernementales (Conseil supérieur de l'Éducation, Conseil des universités, le programme Formation de chercheurs et action concertée (F.C.A.C.)), à la fois relais et tampons de l'intervention étatique dans le système universitaire québécois.

Ces transformations du cadre institutionnel de la fonction universitaire s'accompagnent au début des années 1960, d'une expansion exceptionnelle du nombre de professeurs. En effet, leur effectif double ou presque dans les premières années de la décennie de 1960, passant de 2350 en 1960-61 à 4580 en 1965-66. Par la suite leur nombre continue à augmenter régulièrement mais à un rythme moins rapide jusqu'à la dernière moitié des années 1970. Alors se font sentir nettement les effets de la crise sur le système universitaire et la croissance numérique du corps enseignant est quasi stoppée. 
Si on ne peut nier, derrière ces grandes transformations, l'action d'un facteur démographique résultant du baby boom d'après-guerre et de la montée des aspirations scolaires, il reste bien évident qu'en dernière analyse, tant l'acceptation d'une intervention plus directe et décisive de l'État dans le champ universitaire que la croissance accélérée du corps universitaire renvoient à des stratégies nouvelles que plusieurs groupes sociaux élaborent dans leurs mouvements de reproduction. L'enquête Fortin-Tremblay (1964) n'a-t-elle pas révélé pour la fin des années 1950 des aspirations quasi débridées, chez de larges couches sociales, à une formation universitaire pour leurs enfants?

Si le début de période est signalé par un changement des rapports État-université et l'explosion scolaire, la fin de période nous semble devoir s'établir en 1976 soit au moment où le processus de syndicalisation a atteint, du moins dans le secteur francophone, l'ensemble du corps professoral. Ce processus a transformé non seulement le statut du professeur dans ses rapports à son institution mais aussi l'image qu'il se faisait traditionnellement de lui-même. Dans ce sens-là il s'agit d'un événement majeur.

A travers la conjoncture qui a marqué le développement du système universitaire au cours de cette quinzaine d'années, il s'agira de voir ce qu'il advient du projet humboldtien, d'une fonction universitaire axée sur la recherche. Seront montrés alors la difficulté de réalisation de ce projet, ainsi que les débats renouvelés sur la place de l'enseignement et des services à la collectivité. Par ailleurs, on indiquera brièvement comment se resituent les professeurs suite aux transformations de la structure du pouvoir dans le champ universitaire.

\section{b) Le projet de professionalisation : l'idéal de la recherche, la réalité de l'enseignement}

Si l'explosion scolaire et le financement assuré des universités par l'État favorisaient la multiplication des postes de professeurs dans l'enseignement supérieur et facilitaient la poursuite d'une carrière dans des conditions de revenus mieux assurés et plus élevés, cette situation nouvelle garantissait-elle la réalisation du projet des dernières décennies voulant que la recherche devienne la pratique centrale et distinctive de la fonction universitaire?

On peut en fait se demander dès le départ si la rapidité même à laquelle a dû se faire le recrutement de nouveaux professeurs n'a pas compromis le mouvement de professionnalisation engagé dans les années 1940 et 1950. En dix ans attirer plus de 3000 candidats à des postes de professeur n'est pas chose facile dans une société où l'enseignement supérieur n'était déjà pas très développé.

Dans l'état actuel des connaissances, on ne dispose pas de toutes les informations nécessaires pour établir quelles étaient les caractéristiques précises de ces nouveaux professeurs. Nous savons cependant qu'une partie des recrues venaient des institutions non universitaires, soit des secteurs public et privé. Maheu et al. (1984, p. 263) ont établi que, pour la période allant de 1945 à 1965, c'était le cas de $61 \%$ des professeurs'de psychologie et de $51 \%$ des professeurs de 
Tableau 1 - Evolution des effectifs professoraux dans les universités du Québec, de 1 'Ontario et du Canada, 1960-1980

\begin{tabular}{rrrr}
\hline & Québec & Ontar1o & Canada \\
\hline $1960-61$ & 2350 & 2555 & 7760 \\
$1965-66$ & 4580 & 4695 & 14370 \\
$1970-71$ & 5608 & 9306 & 24612 \\
$1975-76$ & 6732 & 12290 & 30784 \\
$1980-81$ & 7536 & 12865 & 33299 \\
\hline
\end{tabular}

Source : Statistique Canada, Portrait statistique de l'enselgnement supérieur au Canada, 1983, Cat. 81-Y-502F, Tableau 24, P. 52

biologie. Cette étude ne précise cependant pas si ce modèle de recrutement a été celui qui a marqué spécifiquement la période qui nous occupe, soit celle qui débute avec les années 1960. Quoi qu'il en soit, on peut supposer avec vraisemblance qu'une proportion importante des nouveaux candidats ne provenait pas des milieux universitaires. De même on peut supposer qu'une majorité de professeurs ne possédait pas de doctorat si on pense qu'en 1972 il n'y avait encore que 52,3\% et en 1982, $60 \%$ des professeurs qui détenaient un diplôme de $3^{\mathrm{e}}$ cycle (voir tableau en annexe). Il faut ici rappeler cependant que ces faibles pourcentages sont attribuables avant tout aux départements et facultés professionnels qui sont plus près des savoirs pratiques.

Ces quelques indications n'impliquent pas nécessairement que les dispositions vis-à-vis les activités de recherche n'étaient pas fortement valorisées. Les analyses sur le développement des sciences au Québec (dont l'étude de Maheu et al. (1984)) montrent comment, au cours de la période qui nous intéresse, l'activité scientifique s'est institutionnalisée dans le champ universitaire ${ }^{3}$. Un exigence s'impose progressivement comme une norme, celle d'établir comme pré-requis au poste d'universitaire, le diplôme de doctorat ou à tout le moins des études doctorales complétées. Si on est allé chercher des compétences dans la fonction publique et l'entreprise privée, il reste que le recrutement s'est aussi fait auprès de jeunes engagés dans des études doctorales et auprès d'universitaires étrangers. Encore en 1972, pour donner un indice grossier de l'apport de chercheurs étrangers, $23 \%$ des professeurs des universités francophones et $40 \%$ des professeurs des universités anglophones du Québec n'avaient pas la citoyenneté canadienne (M.E.S.S., 1986, Caractéristiques socio-démographiques, p. 14). Maheu et al. (1984) ont observé de leur côté que pour la période 1965-1977, 27 \% des postes de biologistes et $17 \%$ des postes de psychologues sont tenus par des universitaires venant d'autres pays.

Tant dans le cas des jeunes chercheurs engagés dans des études doctorales que dans le cas de professeurs étrangers, il s'agit de recrues socialisées aux valeurs de l'activité scientifique et qui sans doute envisagent dans cette perspective leur carrière universitaire. Il est par ailleurs difficile de reconstituer cette époque sans 
tenir compte de l'élan et du militantisme du noyau de professeurs-chercheurs constitué dans l'après-guerre, professeurs qui ont incité leurs meilleurs étudiants à poursuivre une carrière scientifique en milieu académique ${ }^{4}$. Ce mouvement d'auto-reproduction, qui n'est pas sans analogie avec celui observé, par exemple, dans la constitution et le développement du corps clérical (voir Dandurand, 1988), va contribuer à une autonomisation du champ universitaire. C'est-à-dire que de plus en plus les nouveaux membres viendront d'institutions d'enseignement, passant directement des fonctions d'étudiant à celle d'enseignant. En 1985 seulement $14 \%$ des professeurs avaient occupé des emplois hors du secteur de l'enseignement avant leur engagement à l'université (Crépuq, 1988, p. 18).

La présence de chercheurs chevronnés, celle de jeunes chercheurs sortant à peine d'études doctorales, l'apport d'universitaires étrangers porteurs de traditions de recherche bien établies, tout cela pouvait renforcer le mouvement vers la valorisation de la recherche. Cependant, en dehors même du fait que les besoins du moment ont sans doute obligé à accepter des candidats d'une formation relativement plus faible et moins académique, certaines conditions de la croissance des universités rendaient en elles-mêmes peu facile la réalisation des aspirations à un nouveau modèle d' institutionnalisation du métier d' universitaire axé sur le volet recherche.

En premier lieu, l'engagement hâtif des jeunes recrues signifiait souvent que celles-ci devaient poursuivre leur thèse de doctorat parallèlement à leur tâche d'enseignement et d'encadrement des étudiants. Cette situation limitait leur activité de recherche à leur thèse. Bien plus, le temps et les énergies considérables que supposent les premières préparations de cours et l'apprentissage de la pédagogie, réduisaient d'autant leur participation possible à des activités de recherches. Si en 1960 il y avait 32,3\% des professeurs ayant moins de 35 ans (Fapuq, 1987), en 1971 ce pourcentage est passé à $37,7 \%$ soit plus du tiers (voir tableau 1 en annexe).

Deuxièmement, le support à la recherche, l'accès à des organismes subventionnaires, l'organisation de la recherche elle-même demeuraient encore dans les années 1960 fort limités (v.g. la création du fonds F.C.A.C. se fera seulement en 1969) et tout particulièrement le réseau des universités francophones demeurait sous-développé à cet égard.

Troisièmement, les vagues de nouveaux étudiants déferlaient toujours plus nombreuses sur les universités. La montée des effectifs universitaires était fulgurante et forçait toute l'organisation, mais en particulier elle alourdissait la tâche des professeurs, transformant les conditions mêmes de leur enseignement qui devenait, au premier cycle, un enseignement de masse. La création de l'Université du Québec en 1969, qui sera au départ essentiellement une université de $1^{\text {er }}$ cycle, maintient, dans le réseau francophone, l'attention sur la mission d'enseignement des universités plutôt que sur leur mission de recherche.

Quatrièmement, non seulement sous l'effet des grands nombres, mais aussi sous l'impact des nouvelles sciences du comportement qui sensibilisent certaines couches d'étudiant(e)s à des perspectives plus critiques sur les rapports sociaux, 
l'attention tend à se porter sur la pédagogie. C'est là, comme on le sait, un des aspects de la crise étudiante des années 1960, qui requestionne les modes traditionnels de transmission des connaissances.

En dernier lieu, les années 1960 ont vu les universités s'engager dans d'importantes modifications de leurs structures de gestion, modifications exigées par des transformations des rapports de force tant internes qu'externes au champ universitaire. Ce remue-ménage dans la structure interne du pouvoir qui passe par une laïcisation du corps des officiers supérieurs, par un mouvement accéléré de bureaucratisation des fonctions administratives et par le début du mouvement de syndicalisation des professeurs, va donc solliciter l'attention et drainer une partie des énergies des membres du corps universitaire vers d'autres objets que la recherche.

Ce rappel sommaire de quelques axes importants de l'évolution des universités québécoises durant les années 1960 (rappel qui ne tient même pas compte de toutes les attentes de la société à l'égard du milieu uniiversitaire) suffit à montrer les demandes diverses qui s'exerçaient sur un corps enseignant comprenant une très importante proportion de jeunes. Quand on regarde cette situation avec le recul du temps, on a le sentiment que le milieu universitaire a été coincé entre d'une part des aspirations allant dans le sens d'un statut de distinction lié à une pratique valorisée de recherche, et d'autre part les impératifs immédiats d'assurer l'enseignement aux masses des étudiants du $l^{\text {er }}$ cycle et aussi, bien que secondairement, la nécessité d'investir dans les projets de restructuration administrative et/ou dans leur organisation collective, particulièrement dans le mouvement de syndicalisation. Même si, selon plusieurs analyses (Duchesne, 1978; Fournier, 1986; Maheu et al., 1984; Chartrand et al., 1987) c'est à peu près à la même époque que s'instituent vraiment et que "s'académisent" les pratiques scientifiques au Québec, il reste que le poids de l'enseignement, pour les raisons invoquées plus haut, demeure très lourd et continue à marquer profondément la tâche des universitaires. Mais, d'une certaine façon, sans qu'on en reconnaisse l'entière réalité. En fait tout se passe comme si on résistait à cette réalité et que l'attention était volontairement portée sur l'idéal du chercheur-professeur et d'une université humboldtienne alors que les aspects pédagogiques de la fonction devaient eux demeurer dans l'ombre. D'où des tensions très grandes entre ces deux pôles de la pratique universitaire : l'enseignement et la recherche.

Au cours de la dernière partie des années 1960, suite à la remise en cause par les étudiants des rapports et modes pédagogiques en usage, suite également à une redéfinition-polarisation des rapports professeurs-administrateurs liée au mouvement de syndicalisation du personnel enseignant qui se développe précisément durant ces années, la dimension enseignement revient à l'ordre du jour et réoccupe l'espace des discours dans les débats autour de la tâche.

Ainsi en 1967, dans un colloque organisé par l'Association des professeurs de l'Université de Montréal, sera mis en évidence le peu de préparation et le peu d'intérêt des professeurs et de l'université en général à l'égard de la pédagogie. 
Guy Rocher souligne alors cette lacune fort significative de la valeur et de l'importance des pratiques d'enseignement aux yeux des universitaires.

“... quelle réflexion, demandait-il, apportons-nous à la pédagogie de notre enseignement, c'est-à-dire à cet art de la transmission du savoir?" Il poursuivait : “... l'université apporte généralement une ignorance voulue, sinon un mépris, pour l'art pédagogique. Nous agissons et continuons d'agir comme si le doctorat et quelques publications garantissaient automatiquement la qualité de l'enseignement universitaire." (Rocher, $1967: 8$ )

Non seulement Rocher rappelle le peu d'intérêt pour les questions pédagogiques, mais il dénonce le postulat même à la base d'une conception humboldtienne de l'université : un bon chercheur est (naturellement) un bon pédagogue. Le "chef-d'oeuvre" marquant la fin de l'apprentissage d'universitaire, se résume en un doctorat et quelques écrits scientifiques. On ne songe pas que la pédagogie aussi pourrait ou devrait faire l'objet d'un apprentissage systématique. Ainsi alors que l'on cherche à s'assurer que les professeurs d'université soient de mieux en mieux formés pour la recherche, on ne se préoccupe aucunement de leur donner une formation pédagogique. Dans le contexte où ils ont été prononcés, les propos de Rocher avaient un caractère provoquant non seulement parce qu'ils dénonçaient cette situation, mais aussi parce que, via la pédagogie, il rapprochait le corps universitaire de la grande masse des enseignants de niveau élémentaire, secondaire et collégial. Le statut des universitaires n'était pas encore assez assuré et peut-être aussi assez "distingué", aux deux sens du mot, pour risquer une telle confusion.

$\mathrm{Au}$ tournant des années 1970 , les discours tenus dans quelques colloques et le contenu d'un rapport du Conseil des universités largement publicisé, L'Université québécoise du proche avenir (Hurtubise, 1973), vont nettement dans le sens des remarques de Rocher : affirmation très claire de la nécessité de revaloriser l'enseignement et de moderniser les pratiques pédagogiques.

Par exemple, dans L'Université québécoise du proche avenir, les auteurs présentent comme des défis importants que les universitaires sachent adapter leur enseignement au courant de la pédagogie active et à l'utilisation des moyens audio-visuels, modes pédagogiques de plus en plus en usage aux autres niveaux d'enseignement (p. 113). Mais surtout, ils souhaitent qu'on brise certains mythes entretenus autour de la tâche des professeurs-chercheurs. Citons-les largement puisqu'il s'agit d'une tentative importante de réajustement du projet de professionnalisation de la tâche.

Il faut, écrivent-ils,

"dénoncer le mythe voulant que tout professeur soit un chercheur, revaloriser la fonction d'enseignement ... et créer officiellement le statut de chercheur universitaire. Il nous semble en effet, poursuivent-ils, que la conception selon laquelle tout professeur se double forcément d' un chercheur est irréelle et nocive ... Elle (cette conception) fait partie de l'héritage et de la tradition de prestige des institutions universitaires. (Mais) elle n'est pas conforme aux faits vécus, à la vie de la majorité des professeurs ... il faudra s'attarder à préciser les composantes normales et possibles de la tâche d' un professeur et à revaloriser la composante enseignement". (Hurtubise, 1973, pp. 113-114) 
Plus loin ils ajouteront ce commentaire très révélateur sur les critères d'embauche : "la formation Ph.D., axée exclusivement sur la formation à la recherche, ne devra plus être considérée, même dans l'idéal, comme critère exclusif' (p. 114).

$\mathrm{Ce}$ texte, qu'il aurait fallu citer plus longuement, est fort riche de sens. On peut tenter d'en souligner les points les plus significatifs pour nous. Il y a d'abord une volonté nette de distinguer les deux fonctions de recherche et d'enseignement, remettant ainsi en question la conception humboldtienne de l'universitaire. Ceci amènera les auteurs du rapport à non seulement distinguer les deux aspects de la tâche mais aussi à souhaiter qu'on prévoie des modulations dans son accomplissement, soit une alternance de périodes de recherche et d'enseignement dans la carrière de professeur. Reconnaissant la spécificité et l'importance de l'enseignement, ils proposent que soient pris en considération, au moment de l'engagement, non seulement les attributs de chercheur du candidat, mais aussi ses capacités d'animation et son intérêt dans la relation pédagogique. Enfin, ils dénoncent une vision des choses qui ne tient pas compte de la réalité des tâches des professeurs. Ils visent ici explicitement le fait que la recherche et l'enseignement de masse sont deux réalités spécifiques qui, comme le soulignait dernièrement Gingras (1987), commandent des savoir-faire spécifiques et des qualités distinctes pouvant ou non se retrouver chez un même individu.

Cette incitation à prendre une bonne mesure de la réalité (tâche "normale et possible") semble aussi pouvoir s'appliquer à la reconnaissance de l'importance de l'enseignement, importance réelle qu'on a mis du temps à avaliser mais dont on prend de plus en plus conscience au début des années 1970. Cette conscience ne s'accompagne cependant pas toujours d'une valorisation de cette composante de la fonction d'universitaire. En réalité, pour plusieurs, la place occupée dans les faits par l'enseignement c'est l'échec de l'idéal, du modèle de carrière et du modèle d'université souhaités. En effet, plusieurs universitaires se plaignent de la lourdeur, du poids qu'a imposé l'enseignement de $1^{\text {er }}$ cycle durant cette période de développement rapide. Par exemple, le recteur Kerwin de l'Université Laval, intervenant dans un colloque organisé par l'association des professeurs de son université en avril 1972, se défendra de vouloir augmenter la tâche d'enseignement en soulignant que :

“... depuis cinq ans, une série de documents, de rapports et de résolutions cherchent à libérer l'Université Laval de sa préoccupation traditionnelle vis-à-vis l'enseignement du premier cycle. Cette libération permettrait à chaque professeur de trouver ici une vie proprement universitaire ..." (p. 33) Il dira plus loin que l'Université Laval, malgré ce qu'on en dit "demeure une université essentiellement de $1^{\text {er }}$ cycle et, en particulier, une école de formation professionnelle..." (A.P.U.L., 1972, p. 33)

Sur 1100 professeurs, seulement 400 dirigent alors des thèses de $2^{\mathrm{e}}$ et $3^{\mathrm{e}}$ cycles. C'est dire que cette situation inquiète un certain nombre de professeurs et d'administrateurs qui voient la mission "recherche" des universités compromise par la place occupée par l'enseignement du $1^{\text {er }}$ cycle et la formation professionnelle. Cela est particulièrement ressenti à un moment où commencent à apparaître 
des restrictions budgétaires dans le secteur de l'éducation : comme on le sait, la réforme scolaire cède alors le pas à la réforme dans les domaines de la santé et du bien-être.

Ainsi donc, au terme de cette période, d'un projet de constitution et de professionnalisation du corps professoral principalement axé sur la recherche, on en vient par la force des choses à une vision plus polyvalente de la fonction universitaire. On tente de revaloriser le volet enseignement et de contrer un modèle humboldtien de carrière, modèle normatif qui violente la réalité d'un système universitaire engagé dans un processus de croissance extrèmement rapide et où, bien que les enseignements de $2^{\mathrm{e}}$ et $3^{\mathrm{e}}$ cycles se soient développés, les grandes vagues successives de nouveaux étudiants ont été massivement absorbées dans les programmes de $\mathrm{l}^{\text {er }}$ cycle. Ainsi sont posés les principaux termes des débats autour du processus d'institutionnalisation et de professionnalisation de la fonction universitaire. Ces termes n'ont pas beaucoup varié jusqu'à présent, bien qu'un troisième volet de la tâche, jusqu'alors plus ou moins implicite, se soit entretemps défini et imposé.

\section{c. Service à la collectivité, éducation permanente et mouvements de désinsti- tutionnalisation des savoirs}

C'est au tournant des années 1970 que se définit et se formalise un autre volet de la fonction attribuée au professeur : le service à la collectivité. Selon le Rapport Angers sur l'université (1979), ce volet apparaît dans les documents officiels en 1973. S'ajoutant à l'enseignement et à la recherche, ce sera là un volet de la tâche qui s'institutionnalisera et désormais balisera les fonctions attendues du professeur d'université.

Il est bien évident que sous une forme ou une autre les liens et échanges université/société ont toujours existé. Cependant selon la logique même du processus de professionnalisation dans lequel était alors engagé le corps universitaire, il fallait s'attendre à ce que ces fonctions effectives soient au moins partiellement instituées, c'est-à-dire reconnues, nommées et normalisées.

Le paradoxe est que cette institutionnalisation s'est faite dans le champ universitaire québécois sous l'action de mouvements socio-culturels importants qui réclamaient plutôt une désinstitutionnalisation et une déprofessionnalisation des savoirs. En effet, l'université à la fin des années 1960 et au début des années 1970 est au coeur de la lutte que mènent certains groupes pour faire valoir de nouveaux modèles culturels : tant le mouvement contre-culturel que les promoteurs de l'idéologie de l'éducation permanente demandent que soit en quelque sorte "déclôturé" le champ universitaire, que les connaissances emmagasinées dans l'université s'ouvrent à la collectivité, que les savoir-faire professionnels soient rendus plus accessibles et démystifiés. En fait le discours sur l'éducation permanente, alimenté par les positions de l'Unesco dans le maître-livre, Apprendre à être (Faure et al., 1972) et dans le développement des propos sur l'éducation récurrente autour de l'O.C.D.E., va occuper une place importante, 
dominante même, dans les formulations du projet scolaire du début des années 1970. Entre autres le rapport déjà cité du Conseil des universités, L'université québécoise du proche avenir (Hurtubise, 1972), est profondément marqué par cette problématique. Notons au passage que le discours autour de l'éducation permanente va en quelque sorte prendre au Québec la relève du discours humaniste du Rapport Parent.

C'est donc en ces termes et selon cette orientation idéologique que s'institutionnalise, dans les premiers temps, le volet service à la collectivité. Dans le climat d'alors cela se voulait donc une large ouverture sur le milieu mais, en particulier, une présence mieux assurée aux groupes sociaux les plus démunis et aux clientèles d'adultes qui n'avaient pas eu l'opportunité de poursuivre des études avancées.

Il s'agit là d'un moment de requestionnement important favorisé par la vivacité des grands mouvements socio-culturels de l'époque. Cependant si une certaine tendance en faveur d'une désinstitutionnalisation et déprofessionnalisation de la production-diffusion des connaissances atteint bien le corps universitaire, celle plus lourde d'une institutionnalisation toujours accrue de ces processus demeure déterminante. C'est du moins ce que la situation des dernières années laisse croire : les services à la communauté, entendus au sens généreux du début des années 1970, se rétrécissent comme une peau de chagrin. Maintenant on parle plutôt de renforcer les liens avec l'industrie; le Conseil des universités reproche aux universités de trop développer des programmes courts destinés aux adultes et de dévaloriser ainsi l'enseignement de niveau universitaire. Mais encore plus profondément tout semble s'être passé comme si le rayonnement scientifique avait eu progressivement tendance à occuper le maximum d'espace, mettant au second rang les autres modes de présence au milieu. A ce mouvement, seuls dérogent apparemment certaines universités régionales, dont le statut universitaire est d'ailleurs questionnés.

Voulant justement spécifier ce changement qui s'est opéré chez les universitaires québécois, le Rapport Angers, en 1979, le décrivait comme le passage du statut d'intellectuel au statut d' expert :

“Aujourd'hui, y écrivait-on, le professeur communique habituellement ses connaissances à un public limité de pairs et d'étudiants; et lorsqu'il transmet ses connaissances en dehors de ce cercle de pairs et d'étudiants, il le fait soit à titre d'expert, soit directement par voie de vulgarisation." (Rapport Angers, 1979, livre deuxième, p. 9)

Ainsi les pratiques universitaires, même si elles continuent de différer de façon importante (par exemple selon les champs disciplinaires et les domaines de pratiques professionnelles qui y correspondent) s'inscrivent de plus en plus dans le cadre limité du statut d'expert et la communauté scientifique, semble-t-il, tend à se substituer à la communauté tout court.

Pourquoi cette inclinaison à l'enfermement? Bien sûr parce qu'une ouverture large, trop large, pose des enjeux fondamentaux autour de l'appropriation et du monopole des connaissances, enjeux impliquant au premier chef les institutions de haut savoir et les professeurs. Mais surtout parce que là comme ailleurs un système 
d'échange autour de valeurs centrales détermine les stratégies des acteurs : selon les normes de l'académie, les services à la collectivité contribuent généralement beaucoup moins à la stature scientifique, à la confirmation du rôle d'expert que toute autre forme de reconnaissance par la communauté scientifique elle-même. Et c'est cette stature scientifique, tout au moins dans ses formes externes de reconnaissance (honneurs, titres, publications dans les revues prestigieuses, etc.) qui est à la source même du statut d'universitaire. C'est la monnaie forte et on comprend que les agents du système universitaire, qu'il s'agisse d'individus, de groupes ou d'institutions, auront tendance à miser sur des échanges dans les réseaux scientifiques plutôt qu'au titre de service à la communauté.

Ainsi ce troisième volet, bien qu'il se soit lui aussi formalisé et institutionnalisé pour devenir un des axes de la définition formelle de la tâche, n'aura jamais l'importance du volet recherche. Ni du volet enseignement, ne serait-ce que par le temps qu'on doit y consacrer ${ }^{6}$. De plus, tout porte à croire que sa place deviendra, dans sa forme de service à la collectivité entendu comme la présence aux milieux les plus démunis, encore plus réduite avec la crise financière et le resserrement que connaîtra le système universitaire au tournant des années 1980. Mais tout compte fait, au début des années 1970, avec ce discours sur la nécessité d'ouverture à la collectivité, avec le mouvement de revalorisation de l'enseignement dont nous avons parlé, l'attention semble se décentrer par rapport aux strictes activités de recherche. Le projet des années 1940 et 1950 axé sur la recherche comme pratique distinctive a fait place à une conception plus polymorphe de la fonction universitaire.

\section{d. Position nouvelle des professeurs dans la structure du pouvoir universitaire}

Au cours de la période 1959-1976, à travers les débats et sous l'effet de la conjoncture, on a donc pu voir se modifier, se réajuster mais aussi se préciser les paramètres de la fonction universitaire. Il nous faut maintenant aborder un autre aspect tout à fait essentiel dans le développement du corps professoral : sa position dans la structure du pouvoir, c'est-à-dire plus précisément sa position vis-à-vis l'administration universitaire et, en dernière instance, par rapport à l'État.

De ce que nous avons exposé auparavant, on pourrait dégager deux principes de la constitution in se du corps universitaire, principes qui constituent aussi deux sources de légitimité et les bases, les justifications de leur revendication d'autonomie et de pouvoir : (1) l'appartenance à des disciplines scientifiques, appartenance rappelée constamment par la pratique revendiquée sinon effective de la recherche; (2) l'enseignement qui, s'il constitue un travail important de la diffusion de connaissances, a peut-être d'abord comme signification sociale auprès du public d'être générateur de statuts sociaux fortement recherchés. Cette double source de légitimité lui permet de négocier une certaine autonomie. Mais la marge de manoeuvre laissée au corps universitaire est demeurée relativement précaire dans le mouvement de passage, dans le réseau francophone, d'une université de notables, contrôlée par l'Église catholique, à une université laïque, 
largement financée par l'État et formant des experts de tout ordre. Même si la gouverne universitaire, selon le mode paternaliste des années 1950 [dont témoignent abondamment les récits sur la vie universitaire du temps : voir par exemple les témoignages du Père Lévesque O.P. (1983), ceux de Marcel Trudel (1987), ceux autour de la carrière de Maurice Séguin (Comeau, 1987)] tend à s'estomper en même temps que les structures de gestion se transforment? ${ }^{7}$.

Au cours de la période 1959-1975, il y a d'abord décléricalisation du sommet de la structure du pouvoir dans les universités francophones et l'accession au rectorat des premiers laïcs issus du corps professoral. Il s'agit évidemment là d'une transformation très importante mais une transformation qui ne fait qu'entériner un mouvement déjà bien engagé. En particulier, au tournant des années soixante, la lutte réussie contre le projet d'université des Jésuites avait indiqué le pouls des professeurs vis-à-vis le contrôle clérical de l'enseignement supérieur ${ }^{8}$.

On pourrait penser que durant ces années, marquées d'une part par le déclin clérical et d'autre part par l'état encore embryonnaire du corps administratif, le pouvoir des professeurs aurait été à son maximum. Autrement dit on aurait pu faire l'hypothèse d'un vide momentané du pouvoir, profitant aux professeurs. Hypothèse séduisante peut-être mais qui a peu de chance de correspondre à la réalité. Et cela, pour des raisons qui correspondent à l'état de ce corps professoral. Il est d'abord très divisé, très morcelé, comme nous l'avons déjà signalé. Les ordres de connaissances qu'on privilégie ne sont souvent pas les mêmes, ils sont parfois même en état d'opposition : les cultures scientifiques et professionnelles ne font pas nécessairement bon ménage. Il y a aussi, dans le champ universitaire, de multiples luttes de concurrence entre disciplines pour s'assurer une position avantageuse dans les partages des ressources. Ces divisions sont structurelles et persistent évidemment. Par ailleurs, rappelons qu'au début des années 1960 le corps professoral a absorbé une masse de nouveaux membres. Si cette arrivée donne du poids au groupe et constitue un facteur de dynamisme, il reste qu'il faudra intégrer ces jeunes et surtout qu'entre ces jeunes et les professeurs plus anciens, le mode de regroupement et la position à prendre quant à la gestion de l'université diffèrent.

On peut en avoir un exemple assez net à l'occasion d'un sondage réalisé à l'Université de Montréal en 1967. A la question de savoir s'ils étaient favorables ou non à la syndicalisation, les résultats selon les rangs académiques ont été les suivants :

Proportion de professeurs favorables à la syndicalisation :

- Titulaires : $10,5 \%$

- Agrégés :

$10,8 \%$

- Assistants : $40,2 \%$

- Chargés d'enseignement : 50,2\%

(Source : Résultats d'une enquête de l'A.P.U.M. cité par Fournier, 1977, p. 32)

D'autre part, comme indication des clivages et divisions en fonction de l'appartenance disciplinaire, la répartition des professeurs inscrits au Syndicat des professeurs de l'Université de Montréal en 1972 est aussi significative : $70 \%$ des 
professeurs des sciences humaines et sociales sont membres alors qu'on n'en compte que $48 \%$ en sciences, $11 \%$ en médecine et $2 \%$ en droit (Fournier, 1977, tableau 2, p. 37).

Si le corps universitaire est divisé, il a quant même obtenu une participation au pouvoir mais une participation qui lui attribue le plus souvent un pouvoir purement consultatif. Sa relative autonomie, il la trouve surtout au niveau des regroupements départementaux où les professeurs conservent une certaine zone de responsabilité et de contrôle sur l'enseignement et la recherche.

$\mathrm{Au}$ début de cette période, tout se passe comme si une conjoncture favorable avait contribué à masquer, par le biais d'un ensemble de mécanismes de brouillage, les rapports entre les professeurs et les instances de pouvoir et de contrôle, instances allant de l'administration universitaire aux multiples corps intermédiaires (la Crépuq établie en 1959, le Conseil des universités en 1967, le Conseil supérieur de l'éducation et le ministère de l'Éducation, en 1964). La "conjoncture favorable", c'est d'abord le fait que le corps universitaire cesse toute soumission au clergé à travers la hiérarchie religieuse. C'est aussi le fait que momentanément de très larges secteurs de la population appuient le développement de l'enseignement et en particulier de l'enseignement supérieur : la société québécoise d'alors nourrit en effet un culte de la compétence et adhère, semble-t-il, à cette conception d'un humanisme scientifique qui a trouvé son expression dans le Rapport Parent avec un enthousiasme aussi grand que la distance prise vis-à-vis des croyances religieuses.

A ce prestige de l'universitaire, du fait que les canaux de mobilité, avec le départ de l'Église, s'ouvrent aux laïcs (et aux religieux laïcisés : ce sont eux qui, encore pour un moment, ont reçu le meilleur "in-training" dans le fonctionnement des bureaucraties), s'ajoutent le caractère rotatif de l'occupation des postes administratifs par des professeurs et les ouvertures que permet le contexte d'organisations en pleine expansion. Tout cela contribue à maintenir un brouillage, un flou, entre les frontières de l'administration et les positions de la grande masse des professeurs. Ce sera l'un des obstacles que devra surmonter le mouvement de syndicalisation : amener les professeurs à prendre conscience qu'ils sont des salariés au service d'une institution et non des membres plus ou moins égaux d'une corporation; bien plus, les amener à réaliser qu'ils sont, au même titre que d'autres, des fonctionnaires de l'État provincial. Cette dure réalité, plus difficile à admettre pour ceux qui ont connu l'université d'ancien régime, s'affirmera évidemment à partir de la création du ministère de l'Éducation en 1964.

S'adressant aux professeurs de l'Université de Montréal, Guy Rocher, dans un texte déjà cité et daté de 1967 , cherche justement à rappeler que la situation du professeur d'université a changé considérablement, que l'universitaire est maintenant un professeur comme les autres, 'c'est-à-dire comme la masse des enseignants du secteur public devenu, avec l'étatisation du champ scolaire, quasi directement dépendant de l'État. "Nous sommes irrémédiablement destinés, lance-t-il, au statut de fonctionnaire; qu'on nous le reconnaisse officiellement ou non, cela ne changera pas la réalité." (Rocher, 1967, p. 10). Il exhorte donc les 
professeurs à reconnaître cette nouvelle identité et à se syndiquer pour éviter que les décisions qui les concernent ne se prennent ni au-dessous, ni au-dessus d'eux.

$\mathrm{Si}$, pour défendre les conditions de travail et la place des professeurs dans l'université, l'idée de se replier vers la formule syndicale semble incongrue même souvent inconcevable aux yeux de certains et surtout aux yeux des vieux professeurs dans les vieilles universités, elle est par contre rapidement mise de l'avant par les jeunes professeurs, et particulièrement par les jeunes enseignants d'une université toute nouvelle, l'Université du Québec créée en 1968.

A travers des luttes souvent difficiles (premiềre convention collective signée à l'UQAM en 1971 par un syndicat de professeurś d'université, grèves notamment à Laval et à l'UQAM en 1976), et une dizaine d'années après la création du ministère de l'Éducation, soit en 1975, toutes les universités du secteur francophone voient leurs professeurs réunis dans des syndicats accrédités (Un collectif d'universitaires, 1977; Dégagné et Miller, 1975). Ces syndicats sont soit rattachés à des grandes centrales syndicales, soit regroupés dans la Fédération des associations de professeurs des universités du Québec.

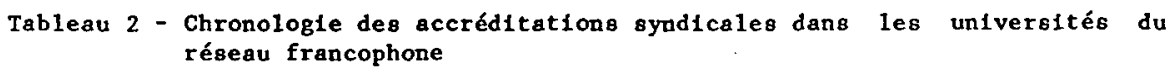

Affiliation

\begin{tabular}{lll}
\hline UQAM & Janv. 1971 & CSN \\
UQTR & Fév. 1971 & Entente de service CSN \\
UQAC & Janv. 1972 & CEQ \\
UQAR & Fév. 1973 & - \\
INRS & Ma1 1973 & Entente de service CEQ \\
U. de Montréal & Ju11. 1975 & FAPUQ \\
U. de Sherbrooke & Fév. 1974 & FAPUQ \\
U. Lava1 & Janv. 1975 & FAPUQ \\
Polytechnique & Fév. 1971 & FAPUQ \\
\hline
\end{tabular}

Source: Un collectif d'unfversitalres, Le syndicalisme universitalre et 1'État, Montréal, HMH, 1977, tableau 1, pp. 8-9.

Le mouvement de syndicalisation a été relativement rapide malgré les résistances et malgré le brouillage des frontières entre administration et professeurs. Il marque certes un changement majeur dans l'identité des professeurs mais, en même temps, il ne solutionne pas tous les problèmes que soulève leur position dans la structure du pouvoir de l'université, loin de là. Avant de revenir sur ce point, arrêtons-nous un moment à resituer le contexte qui prévaut au moment de ce processus de syndicalisation.

Avec la création du ministère de l'Éducation et sa Direction de l'enseignement supérieur, l'État devient non seulement le pourvoyeur financier de l'enseignement supérieur mais aussi une instance importante de planification. Le prolongement de son action, avec la création de l'Université du Québec en 1968, affermit considérablement sa position à l'intérieur même du système universitaire. Il a en quelque sorte deux solides points d'appui pour intervenir : l'un, plus extérieur, la 
Direction générale de l'enseignement supérieur du ministère de l'Éducation (DGES), l'autre plus interne, le réseau de l'Université du Québec. Présence donc très concrète de l'État qui fera craindre aux professeurs une perte de contrôle sur leurs conditions de travail, comme on l'a souligné. C'est cette crainte par exemple que l'on sent clairement dans les propos tenus en 1972 à un colloque des professeurs de Laval (A.P.U.L., 1972). Circule à ce moment-là la rumeur que le ministère pense à imposer aux professeurs une charge plus lourde d'enseignement. Même menace que le Rapport Gobeil, quinze ans plus tard, fera à nouveau planer. Donc appréhension d'une perte d'autonomie et de contrôle sur les conditions d'exercice de la tâche. Cette menace, elle vient aussi d'une administration plus présente, avec la bureaucratisation croissante du système universitaire, et d'une administration qui souvent, aux yeux des professeurs, joue le rôle de simple relais du ministère ${ }^{9}$. Enfin, un dernier élément est important : le militantisme étudiant de la fin des années 1960 prend comme cible notamment le pouvoir des professeurs. Cette conjoncture a pour effet de placer les universitaires dans une position de défense. De toute part, semble-t-il, leur place et leur autonomie sont questionnées, remises en cause.

Un des enjeux majeurs que pose l'action syndicale au niveau des structures concerne justement le droit de gérance que revendique l'administration et qui s'appuie sur la nécessité fonctionnelle d'instances décisionnelles qui doivent orienter l'institution universitaire dans son ensemble et opérationnaliser ses orientations dans et à travers des structures complexes, face aux intérêts multiples et souvent divergents de ses agents. En fait, le caractère éclaté, différencié des préoccupations et orientations des agents de l'institution, en particulier chez les professeurs, devient l'une des sources de légitimité et de pouvoir des administrateurs d'université.

Dans le mouvement de professionnalisation du corps universitaire, la syndicalisation peut apparaître comme une avancée mais une avancée qui par certains côtés limite le champ d'action du groupe. C'est une avancée dans la mesure où les professeurs se sont donnés, par cette voie, un moyen efficace de maintenir un certain contrôle sur leurs conditions de travail, soit sur les salaires, les congés, les pensions, mais aussi sur les conditions d'entrée, les critères et procédures de promotion, la charge de travail. Par ailleurs, le type de relation que le mode d'action syndicale instaure avec l'administration s'articule plus ou moins bien à la participation des professeurs aux instances de décision : cette participation demeure ambiguë. ${ }^{10} \mathrm{Ce}$ que les administrateurs ne manquent pas de faire valoir en montrant par exemple les contradictions que vivent les professeurs qui, comme chefs de département ou membres de comité, sont placés en situation de conflit de rôles. Mais il n'est pas du tout certain que l'administration soit perdante puisque de cette manière les structures de participation permettent d'impliquer les professeurs, en dehors du cadre syndical, dans des décisions qui les concernent. Dans ces circonstances, en utilisant des pouvoirs de nomination et de cooptation, une administration peut être tentée de jouer au ventriloque, c'est-à-dire de recourir aux comités de professeurs pour faire passer ses orientations, ses directives. 
Quoi qu'il en soit, l'ambiguité est là et il n'est pas toujours facile de voir clairement comment elle sert ou non l'une ou l'autre des parties. Cependant il nous semble certain que dans le mouvement de professionnalisation du corps professoral, la syndicalisation se présente comme un outil indispensable de contrôle sur les normes institutionnelles qui balisent de manière de plus en plus serrée les tâches et attributs de la fonction universitaire. On peut évidemment reprocher au syndicalisme d'accélérer ou, du moins, d'avaliser le mouvement de bureaucratisation des universités et d'ajouter aux rigidités du système. Mais on voit mal comment, avec l'étatisation du champ de l'enseignement supérieur et la bureaucratisation quasi inévitable de la gestion des universités, les professeurs pouvaient, par d'autres voies que celle-là, s'assurer d'une place et d'un certain contre-pouvoir.

L'idéologie de la participation, alors si forte en certains milieux québécois et qui s'est matérialisée à l'intérieur du champ universitaire d'abord dans l'expérience historique de l'université du Québec, (Dorais, 1977), mais aussi, à un moindre degré, dans les nouvelles structures de gestion des universités Laval, de Montréal et de Sherbrooke, ce rêve renouvelé de la communauté universitaire s'est terminé, somme toute, par une polarisation plus grande des rapports administrateurs/ professeurs. Ainsi, le ralliement autour d'une conception plus traditionnelle de l'université conçue comme une "communauté" où se fondent dans un tout professeurs et administrateurs, cette image de l'université-grande-famille réunie autour de la figure paternelle du recteur, s'est profondément altérée durant cette période. On assiste à une étape majeure dans la transformation de la culture universitaire. Le professeur, sous l'aura du libre-chercheur, de l'intellectuel sans attache, se découvre simple salarié et même, en dernière instance, fonctionnaire de l'État.

"Demain ne sera plus jamais pareil", écrivait en 1976 Jean-Marc Piotte (Un collectif d'universitaires, 1977, pp. 153-159) pour décrire cette transformation qui s'achève dans les grèves qui, en 1976, vont frapper le milieu universitaire, celle de l'UQAM et surtout celle de Laval, université bien établie et plutôt perçue jusque-là comme traditionnelle et conservatrice. Si ce mouvement de syndicalisation signifie un nouveau mode d'intégration des professeurs à l'université et les force à développer une conscience nouvelle de leur appartenance au groupe qu'ils constituent dans le système universitaire, il faut aussi rappeler que cela n'implique cependant pas une rupture complète avec des conceptions que les professeurs entretenaient sur leur situation. Malgré la polarisation nouvelle entre l'adiministration et le corps professoral, le sentiment d'appartenance à une université et à une "communauté" où les administrateurs sont "des nôtres", s'il n'a plus la force d'antan, continue malgré tout à se manifester. Le recrutement des administrateurs parmi les professeurs, les nombreux comités où siègent ces derniers, même s'il s'agit d'instances uniquement consultatives, sont des situations qui continuent d'entretenir des zones importantes d'ambiguité et d'incertitude et qui permettent de maintenir ce brouillage, dont nous avons déjà parlé, quant à la participation des professeurs au pouvoir universitaire. Par ailleurs, il ne faut pas oublier que 
l'identité première des universitaires s'est construite dans et à travers un travail profond de socialisation à une discipline scientifique ou professionnelle. Tout membre du corps universitaire est aussi chimiste, médecin, anthropologue, ingénieur, etc. et les départements sont généralement constitués sur une base disciplinaire. On conçoit qu'une telle appartenance rende difficile le développement d'une identité spécifique attachée à la fonction de professeur d'université.

A la fin de cette période, le contexte institutionnel dans lequel s'inscrit le corps professoral s'est donc transformé considérablement. En fait on a assisté à la mise en marche de trois processus convergents : une étatisation partielle mais déterminante du réseau des universités québécoises; une bureaucratisation croissante de la gestion universitaire; une syndicalisation rapide du corps professoral, du moins dans les universités francophones. Dans cette conjoncture, on a vu aussi les effectifs professoraux se multiplier sous l'effet de la croissance des clientèles étudiantes et atteindre une masse critique qui, disons-le maintenant, ne se modifiera pas de façon importante par la suite.

Il est bien certain que ces transformations dans le champ universitaire réalisaient les prérequis d'une profession au sens où le rapport Tremblay l'entendait : une sécurité financière pour le professeur et une reconnaissance juridique. La communauté universitaire possède dès lors son "ordre" laïc. On sait cependant que telles n'étaient pas les limites des aspirations professionnelles des universitaires les plus dynamiques. Pour eux, dans l'université nouvelle, le professeur se devait de mettre au centre de ses préoccupations la recherche scientifique. Ainsi le mouvement de professionnalisation engagé dans les années soixante a d'abord été marqué par la tension entre ce projet idéal et la réalité d'une université alourdie par le poids d'une clientèle grandissante. Par la suite, on a assisté à une tentative de revalorisation des tâches d'enseignement et à une plus grande sensibilisation à l'importance des services à la collectivité.

Ainsi, au terme de cette période, non seulement le contexte institutionnel a-t-il subi des transformations majeures mais les grands contours de la tâche et de la "mission" du professeur sont maintenant mieux définis : recherche, enseignement et services à la collectivité. Les débats et enjeux autour de la profession universitaire vont désormais s'inscrire à l'intérieur de ce territoire.

Dans les dix années qui suivent, le mouvement de professionnalisation va s'approfondir. Simultanément les professeurs vont devoir s'engager avec les bureaucraties universitaires dans des luttes serrées autour de normes institutionnelles devenues de plus en plus nombreuses et complexes. La crise accentuera la tendance, déjà présente, à la multiplication des contrôles administratifs, et des critiques sévères à l'endroit du corps professoral [v.g. Rapport Gobeil (1986); Rapport Lacroix (1985)] pourront paraître justifier ces contrôles.

Ainsi, l'élan à la base du mouvement de constitution d'un corps professoral laïc et converti à la rationalité scientifique, mouvement guidé par le modèle de l'expert et accordé à la modernisation du Québec, semble s'évanouir progressivement dans un processus d'institutionnalisation-normalisation des fonctions complexes de 
l'universitaire. Chaque pas nouveau enferme les professeurs dans un nouveau contrôle. L'enseignement et la recherche se font de plus en plus sous surveillance, non pas idéologique mais technocratique, alors même que le corps universitaire québécois n’a jamais été aussi qualifié, ni expérimenté.

\section{NOTES}

1 Cette analyse est d'abord une lecture sociologique du corps professoral des universités francophones du Québec. Elle n'aborde pas de façon systématique la comparaison entre les universités francophones québécoises et les autres universités canadiennes ou américaines. Ceci pourrait être l'objet d'une autre étude. Le lecteur attentif décèlera néanmoins qu'une telle comparaison établirait à la fois des convergences et des divergences.

2 Ces modifications ne sont pas sans lien entre elles car si d'une certaine façon le projet de professionnalisation n'était réalisable que par une étatisation au moins partielle de l'enseignement supérieur, comme dans le cas de nombreux autres groupes professionnels, cette dynamique elle-même a amené les professeurs d'université à une stratégie de défense où le syndicalisme apparaîtra comme la forme institutionnelle la plus susceptible de protéger leurs droits.

On trouve ici un autre exemple montrant que les rapports État-profession sont complexes et que l'intervention étatique ne va pas univoquement dans le sens d'une simple désappropriation des pouvoirs "privés" des professions. Ce que Terry Johnson (1982), parmi d'autres, a justement tenté de mettre en évidence.

3 Voir Duchesne (1978), Fournier (1986), Chartrand et al. (1987).

4 Pour mémoire, on peut rappeler quelques noms : par exemple, en sciences, ceux de Pierre Demers, de Paul Lorrain, d'Armand Frappier, de Pierre Dansereau; en mathématiques ceux d'Adrien Pouliot, de Maurice Labbé; en sciences humaines et sociales, ceux du Père G.H. Lévesque, de Maurice Séguin, Guy Frégault, du Père Mailloux, etc. Cette liste en oublie plusieurs qui, peut-être moins connus, n'ont pas moins incité leurs meilleurs élèves à entreprendre des carrières de recherche. Voir aussi Foumier (1986) sur cette question.

5 Lors de la commission parlementaire de l'automne 1986 sur l'enseignement supérieur, les constituantes de l'université du Québec situées dans des régions éloignées des grands centres ont bien fait valoir l'importance de leur implantation dans leur milieu. Elles ont en particulier tenté de démontrer leur légitimité à partir des services qu'elles rendent à la communauté qui les entoure. Lise Bissonnette, analysant la défense de ces universités, dégageait bien à la fin de sa chronique la place de telles universités dans le champ universitaire. Voici ce qu'elle écrivait :

"Est-ce là l'université de l'avenir? Ou sommes-nous en train de consacrer, en l'encensant, un système universitaire à deux faces? Les universités des grands centres, moins sollicitées, pourront poursuivre la grande mission académique d'enseignement et de recherche qui attirera toujours les élites et leurs enfants; les universités régionales seront celles du quotidien pratique, du tout-terrain, et peut-être bien la "voie allégée", en somme, de l'enseignement universitaire. Quand les universitaires deviennent trop tout à tous, a rappelé discrètement Claude Ryan, ils risquent de ne plus être bons pour "la grosse ouvrage" qui est leur mission première." (Le Devoir, 11 octobre 1986).

6 Les études de budget-temps que nous connaissons (F.A.P.U.Q., 1987; Rapport Lacroix, 1985) rappellent la place prépondérante du temps consacré à l'enseignement, même dans les multi-universités.

7 Cette question de la transformation de la structure interne du pouvoir dans les universités québécoises du réseau francophone mériterait évidemment de faire à elle seule l'objet d'une recherche. Nous ne pouvons ici que l'évoquer, formuler quelques hypothèses et tenter de situer dans ces structures la position du corps professoral. Sur cette question on pourra se référer aux travaux de Crête et al. (1982).

8 Voir la position prise par les professeurs de l'Université de Montréal sur cette question dans L'Université dit non aux Jésuites (A.P.U.M, 1961). L'Association des professeurs de Laval a par ailleurs donné son appui total à l'action de leurs collègues de l'Université de Montréal. 
9 Cette perception du rôle de l'administration sous-tend une partie des textes réunis dans le collectif d'universitaires (1977). Elle apparaît clairement dans les débats sur la tâche organisés par les professeurs de Laval en 1972 (A.P.U.L., 1972).

10 Voici comment la Crépuq présentait la situation nouvelle créée par la syndicalisation des professeurs: "Certes la participation et la consultation des professeurs continuera d'exister, mais en dehors de la convention collective et par le truchement des organismes habituels. La distinction s'établira entre le corps professoral et le syndicat, le premier étant constitué de professionnels comme antérieurement, alors que le second, limité à la négociation de conditions de travail, regroupera des professeurs défendant leurs intérêts propres. L'avenir révèlera dans quelle mesure cette distinction est possible ou chimérique: elle vise en tout cas à établir un nouvel équilibre des forces en maintenant et confirmant les responsabilités de la corporation universitaire et de ses organismes (conseil, assemblée universitaire, commission des études, etc.)" (Un collectif d'universitaires, 1977).

\section{Annexe}

Evolution du corps professoral selon certaines caractér1st1ques 1972-73, 1982-88, 1985

\section{Proportion de profesgeurs détenant un d}
a) établissement d'enseignement
b) établissement étudiant
c) secteur public
d) Industrie/conmerce
e) autres

\section{Répartition (\%) selon le rang académique}

Rang 1/chargé d'enseignement

Rang 3/agrégé

Rang 4/titulaire

Sans rang/autres

\section{Répartition (z) selon l'âge}

\author{
Age moyen \\ Molns de 35 ans \\ 35-49 ans \\ 50 ans et plus
}

\section{Répartition ( $\%$ ) selon les sexes}

Rang 2/adjoint

$\begin{array}{lrr}(1970) 3 & & \\ - & 4,2 & 2,6 \\ 37,8 & 20,0 & 18,2 \\ 24 & 42,9 & 42,2 \\ 15,9 & 29,1 & 34,6 \\ 22,0 & 3,9 & 1,8\end{array}$

$\begin{array}{lll}(1971)_{3} & (1981)_{2} & \\ 37,8 \text { ans } & 42,9 \text { ans } & 45 \text { ans } \\ 37,7 & 16,8 & 11,1 \\ 46,5 & 60,2 & 59,3 \\ 15,8 & 23 & 30,2\end{array}$

Fenme 8

13,7

16,7

16,3

86,3

83,3

83,7

Sources : 1. Caractérlotiques soclo-démographlques et statut des profeseurschercheurs des universitéa du Québec, Miniatère de l'Enselgaement superriour et de la Sclence, Québec, Gouvernement du Québec, 1986, Bulletin statlat1que 86-02.

2. Caractériotiques et rémútration des profesaeurs de carrière des unlversités québécolses, Crépuq, Montréal, 1988.

3. F.A.P.U.Q., Statistiques sur les professeurs d'université, leur tâche et leur environnement de travall, Leo services de recherche éconounlque et sociale, Montréal, janvier 1987. 


\section{BIBLIOGRAPHIE}

A.P.U.L. (Association des professeurs de l'Université Laval) (1972). La charge professorale : une problématique nouvelle, Actes du colloque du 8 décembre 1971, numéro spécial de Forum universitaire, no 10 , avril.

A.P.U.M. (Association des professeurs de l'Université de Montréal) (1961). L'université dit non aux Jésuites, Montréal, Éditions de l'Homme.

Bélanger, P., Daoust, G. (1973). L'université dans une société éducative, Rapport du comité d'étude sur l'éducation des adultes et l'éducation permanente dans les universités du Québec, Crépuq et Conseil des universités.

Bertrand, M. (1985). Les professeurs d'université au Canada ou la profession universitaire, Statistique Canada, section de l'étude sur les professions (A paraître)

Blondin, D. (1987). Les rapports entre l'enseignement et la recherche dans la profession universitaire, Thèse de doctorat, Faculté des sciences de l'éducation, Université de Montréal.

Bourdieu, P. (1984). Homo Academicus, Paris, Editions de Minuit.

Brunet, M. (1963). Le financement de l'enseignement universitaire au Québec, Montréal, L'Académie canadienne-française.

Brunet, M. (1967). Le professeur des universités franco-québécoises, évaluation de son rôle au sein de notre société. Cahiers de Cité libre, no 5, juin, pp. 11-17.

Caplow, T., McGee, R. C. (1961). The Academic Market Place, New York, Sciences Editions.

Chartrand, L., Duchesne, R., Gingras, Y. (1987). Histoire des sciences au Québec, Montréal, Boréal.

Comeau, R. (ed.) (1987). Maurice Séguin, historien du pays québécois, Montréal, V.L.B.

Commission conjointe du conseil et de l'Assemblée universitaire. (1970). L'université, son rôle, le rôle de ses composantes, les relations entre ses composantes, Montréal, Presses de l'Université de Montréal.

Conseil des Universités. (1980). Onzième rapport annuel, 1979-80, Éditeur officiel du Québec.

CRÉPUQ (Conférence des recteurs et des principaux des universités du Québec). (1988). Caractéristiques et rémunération des professeurs de carrière des universités québécoises.

Crete, Jean, Goyete, Line, Laflamme, Jacques, Landry, Réjean, Lemieux, Vincent, Masson, Dominique. (1982). Apercus nouveaux sur les universités du Québec, Série 2, Notes et travaux de recherches no 3, Laboratoire d'études politiques et administratives, Université Laval.

Dandurand, P. (1988). Hégémonie et éducation : Église, École et État dans les rapports de pouvoir au Québec, 1940-1965. Texte inédit, 108 p.

Degagne, A., Miller, R. (1975). L'université et la syndicalisation de ses professeurs, Étude spéciale no 3, Réalisée pour le Conseil des universités, Québec, Éditeur officiel.

Dion, L. (1958). Aspects de la condition du professeur d'université dans la société canadiennefrançaise. Cité Libre, no 26, juillet.

Dorais, L. (1977). L'autogestion universitaire, autopsie d'un mythe, Montréal, Presses de l'Université du Québec.

Duchesne, R. (1978). La science et le pouvoir au Québec (1920-1965), Coll. Études et Dossiers, Ministère des Communications, Québec, Éditeur officiel du Québec.

Dumas, B. (1987). Philosophy and Sociology in Quebec : A Socio-Epistemic Inversion. The Canadian Journal of Sociology, vol. 12, nos 1-2, pp. 111-133.

Falardeau, J. (1959). Lettre à mes étudiants, à l'occasion des vingt ans de la Faculté des sciences sociales de Québec. Cité Libre, no 23, mai, pp. 4-14.

F.A.P.U.Q. (Fédération des associations de professeurs des universités du Québec) (1987). Statistiques sur les professeurs d'université, leur tâche et leur environnement de travail, Les Services de recherche économique et sociale, janvier.

Faure, E. et al. (1972). Apprendre à être, Paris, Fayard Unesco.

Fortin, G., Tremblay, M.-A. (1964). Les comportements économiques de la famille salariée du Québec, Québec, Les Presses de l'Université Laval.

Fournier, M. (1977). Structures universitaires et pratiques syndicales à l'Université de Montréal. In : Un collectif d'universitaires, Le syndicalisme universitaire et l'État, Montréal, $\mathrm{HMH}$, pp. 29-36. 
Fournier, M. (1986). L'entrée dans la modernité. Science, culture et société au Québec, Montréal, Editions Saint-Martin.

Freidson, E. (1986). Professional powers, a study of the institutionalization of formal knowledge, Chicago, University of Chicago Press.

Gingras, Y. (1987). Le défi de l'université moderne : l'équilibre entre l'enseignement et la recherche. Possibles, vol. 11, no 4, pp. 151-163.

Harris, R. (1986). A history of higher education in Canada, 1663-1960, Toronto, University of Toronto Press.

Hughes, E.C. (1971). The sociological eye. Selected papers. Chicago, Aldine Atherton.

Hurtubise, R. (ed.) (1973). L'université québécoise du proche avenir, Montréal, Hurtubise HMH.

Johnson, T. (1982). The State and the Professions : Peculiarities of the British. In A. Giddens, J. McKenzie (eds), Social Classes and the Division of Labor, Cambridge, Cambridge University Press.

Ladd, E.C., Lipset, S. M. (1975). The divided academy, professors and politics. New York, McGraw Hill.

Lévesque, G. (1983). Souvenances I, Montréal, Éditions La Presse.

Lévesque, G. (1984). La première décennie de la Faculté des sciences sociales à l'Université Laval. In G. H. Lévesque et al. (eds), Continuité et rupture : les sciences sociales au Québec, Montréal, Presses de l'Université de Montréal.

Maheu, L. (1981). L'université québécoise francophone et le changement social : un essai en sociologie comparative. Texte miméographié, Montréal, Département de sociologie, Université de Montréal, $50 \mathrm{p}$.

Maheu, L., Descarries-Bélanger, F., Fournier, M., Richard, C. (1984). La science au Québec francophone : aperçus sur son institutionnalisation et sur les conditions d'accès à sa pratique. Revue canadienne de sociologie et d' anthropologie, vol. 21, no 3, pp. 247-274.

Ministère de l'enseignement supérieur et de la science. (1986). Caractéristiques socio-démographiques et statut des professeurs/chercheurs des univesités québécoises en 1982-1983 et évolution depuis 1972-1973, Gouvernement du Québec.

Ministère d'État au développement culturel. (1979). Pour une politique québécoise de la recherche scientifique, Québec, Éditeur officiel.

Ostry, S. (ed.) (1972). Canadian higher education in the seventies, Ottawa, Conseil économique du Canada.

Rapport Angers. (1979). Rapport de la Commission d'étude sur les universités, Québec, Éditeur officiel du Québec.

Rapport Gobeil. (1986). Groupe de travail sur la révision des fonctions et des organisations gouvernementales, Rapport, Gouvernement du Québec.

Rapport Lacroix. (1985). Groupe de travail sur les priorité La poursuite de l'excellence, Montréal, Université de Montréal, Comité de la planification, juillet.

Rapport Massey. (1951). Rapport de la Commission royale d'enquête sur l'avancement des arts, des lettres et des sciences au Canada, Ottawa, Imprimerie du Roi.

Rapport Parent. (1963-1966). Rapport de la Commission royale d'enquête sur l'enseignement dans la province de Québec, Gouvernement du Québec.

Rapport Tremblay. (1956). Rapport de la Commission royale d'enquête sur les problèmes constitutionnels, Province de Québec.

Rocher, G. (1967). Le professeur d'université. Un enseignant pas comme les autres? Cahiers Cité Libre, no 5, juin, pp. 5-10.

Sorecom. (1982). Les Québécois face aux universités et aux universitaires du Québec, Montréal, mai.

Statistique Canada. (1983). Portrait statistique de l'enseignement supérieur au Canada, Des années 1960 aux années 1980 , Édition 1983, Ottawa.

Trudel, M. (1987). Mémoires d'un autre siècle, Montréal, Boréal.

Un collectif d'universitaires. (1977). Le syndicalisme universitaire et l'État, Montréal, $\mathrm{HMH}$. 